\title{
List of angiosperm species in an Atlantic Forest fragment reveals collection gaps in Espírito Santo state, Brazil
}

\author{
Jaquelini Luber ${ }^{1,2}$, Amélia C. Tuler ${ }^{1,5}$, Filipe Torres ${ }^{1,3}$, Jheniffer A. Christ ${ }^{1,3}$, Karlo G. Guidoni-Martins ${ }^{1,2}$, \\ Marcilio Zanetti ${ }^{1}$, Renan K. Hollunder ${ }^{1}$, Vitor C. Manhães ${ }^{1,3}$, João Paulo Fernandes Zorzanelli ${ }^{4}$, \\ Eduardo S. Mendonça ${ }^{6}$, Mário L. Garbin ${ }^{1,6,7}$ and Tatiana T. Carrijo ${ }^{1,2,3 *}$
}

1 Laboratório de Botânica, Centro de Ciências Agrárias, Universidade Federal do Espírito Santo, Alto Universitário, s/n, Guararema, 29.500-000, Alegre, ES, Brazil

2 Programa de Pós-graduação em Biodiversidade Tropical, Centro Universitário Norte do Espírito Santo, Universidade Federal do Espírito Santo, Rodovia BR 101 Norte, Km 60, Bairro Litorâneo, 29932-540, São Mateus, ES, Brazil

3 Programa de Pós-graduação em Genética e Melhoramento, Centro de Ciências Agrárias, Universidade Federal do Espírito Santo, Alto Universitário, s/n, Guararema, 29.500-000, Alegre, ES, Brazil

4 Programa de Pós-graduação em Ciências Florestais, Centro de Ciências Agrárias, Universidade Federal do Espírito Santo, 29.550-000, Jerônimo Monteiro, ES, Brazil.

5 Escola Nacional de Botânica Tropical, Instituto de Pesquisas Jardim Botânico do Rio de Janeiro, Rua Pacheco Leão, 2040, 22.460-030, Horto, RJ, Brazil

6 Programa de Pós-graduação em Produção Vegetal, Centro de Ciências Agrárias, Universidade Federal do Espírito Santo, Alto Universitário, s/n, Guararema, 29.500-000, Alegre, ES, Brazil

7 Programa de Pós-Graduação em Ecologia de Ecossistemas, Laboratório de Ecologia Funcional, Universidade Vila Velha, Rua Comissário José Dantas de Melo, s/n, Boa Vista, 29102770, Vila Velha, ES, Brasil

* Corresponding author. E-mail: tcarrijo@gmail.com

\begin{abstract}
This study presents a list of angiosperm species in an Atlantic Forest fragment in the southern portion of Espírito Santo state, Brazil, a region that represents a collection gap within the Atlantic Forest. The studied site is a relatively small fragment of 144 ha located within a conservation unit, the Mata das Flores State Park. The site belongs to a conservation priority area for the Atlantic Forest in Espírito Santo, and is under strong anthropic pressure. Of the 239 species listed here, 21 are new records for the state, eight are endemic, and 20 figure either in the country's or the state's Red Lists of endangered species. Rubiaceae and Piperaceae were the families with the highest number of species. We show that small fragments that were never inventoried before can reveal a relatively large number of threatened species and that collection gaps need to be filled in order to refine our understanding about conservation priorities within the Atlantic Forest Biome.
\end{abstract}

Key words: checklist; collection gaps; conservation status; new records

\section{INTRODUCTION}

Collection gaps affect the knowledge about species conservation statuses and endemism levels, and hamper effective management planning for conservation areas
(Grand et al. 2007; Joppa et al. 2011). This is of great concern considering the high endemism levels of Southeast Brazil and that most known species have small distribution ranges (Pimm et al. 2014). Fragments with less than 100 ha correspond to $30-40 \%$ of all remnants in the biome (Ribeiro et al. 2009), which has high levels of floristic richness, endangered species, and endemism (e.g., Mori et al. 1981; Peixoto 1992). Most of this knowledge is based on floristic studies and local floras, which can also lead to the description of new species and new occurrences (Baitello 2001; Deble 2005; Deble et al. 2006; Oliveira and Deble 2006; Coelho 2010; Brotto and Baitello 2012). The description and identification of new species are important topics for biodiversity research agendas (see Joly et al. 2014). Moreover, this biome has the highest number of endangered species in Brazil and the southeast portion of the biome houses the majority of these species (Martinelli et al. 2013).

The number of endemic species of an area is directly affected by collection effort (see Murray-Smith et al. 2009; Werneck et al. 2011). It is noteworthy that from the 8,630 endemic species of the Atlantic Forest, 1,104 $(12.8 \%)$ are indigenous from Rio de Janeiro state, whereas the neighboring state of Espírito Santo, with nearly the same total area of Rio de Janeiro, have almost half (551) of this value (List of Species of the Brazilian Flora 2014). Despite the increased knowledge about 
the Atlantic Forest flora (Stehmann et al. 2009; Forzza et al. 2010), some regions of this biome remain poorly known. Studies about centers of endemism have shown a geographical bias of sampling effort in the central and northern portions of the Espírito Santo state (Murray-Smith et al. 2009; Werneck et al. 2011), while the southern portion have insufficient data of species occurrences. Nevertheless, the floristic composition of the remnants will reflect their history, making each remnant unique (Santos and Kinoshita 2003).

Floristic studies are crucial in order to fill these collection gaps. Field surveys are often viewed as the obvious, though expensive, way to do this. In the absence of information about the species richness, alternative approaches, such as mapping of vegetation cover (Ribeiro et al. 2009), landscape structure (Metzger 2000), or predictive models (Bini et al. 2006) can be used as surrogates for the expensive and time consuming field surveys, in order to subsidize conservation strategies. Such approaches have the advantages of covering larger spatial extents and providing faster solutions. However, systematic field surveys are the obvious and best available solution to increase the knowledge about biodiversity (Margules and Pressey 2000). This is because most of these models are based on local inventories, and they suffer with collection gaps and lack of knowledge about the number of new, endemic or endangered species in a given site. Therefore, even though systematic field surveys have several operational shortcomings, they are the definitive solution to reveal how many species a site holds. Here we report the results of an intensive field survey on a 144 ha of an Atlantic Forest remnant within a collection gap in the southern portion of the Espírito Santo state, Brazil.

\section{MATERIALS AND METHODS \\ Study site}

The study was conducted in a 144 ha forest fragment (Figure 1) located in in the Mata das Flores State Park (MFSP; $20^{\circ} 35^{\prime} 54^{\prime \prime} \mathrm{S}, 041^{\circ} 10^{\prime} 53^{\prime \prime} \mathrm{W}$ ), Municipality of Castelo, Espírito Santo state (ES). The area comprises a Lowland Ombrophilous Forest, with mean annual precipitation of $1,200 \mathrm{~mm}$ (Oliveira-Filho et al. 2005), and elevation ranging between $100 \mathrm{~m}$ and $800 \mathrm{~m}$. The park is adjacent to the urban area of Castelo and is surrounded by cattle farms and coffee crops (IEMA 2012). There are evidences of past selective logging within the Park. Nonetheless, the MFSP is an important corridor connected to two other state parks located at higher altitudes, Forno Grande and Pedra Azul.

\section{Floristic study}

Plant samples were collected on a weekly basis from August 2012 to June 2014 through expeditious walks on the studied fragment. Fertile specimens
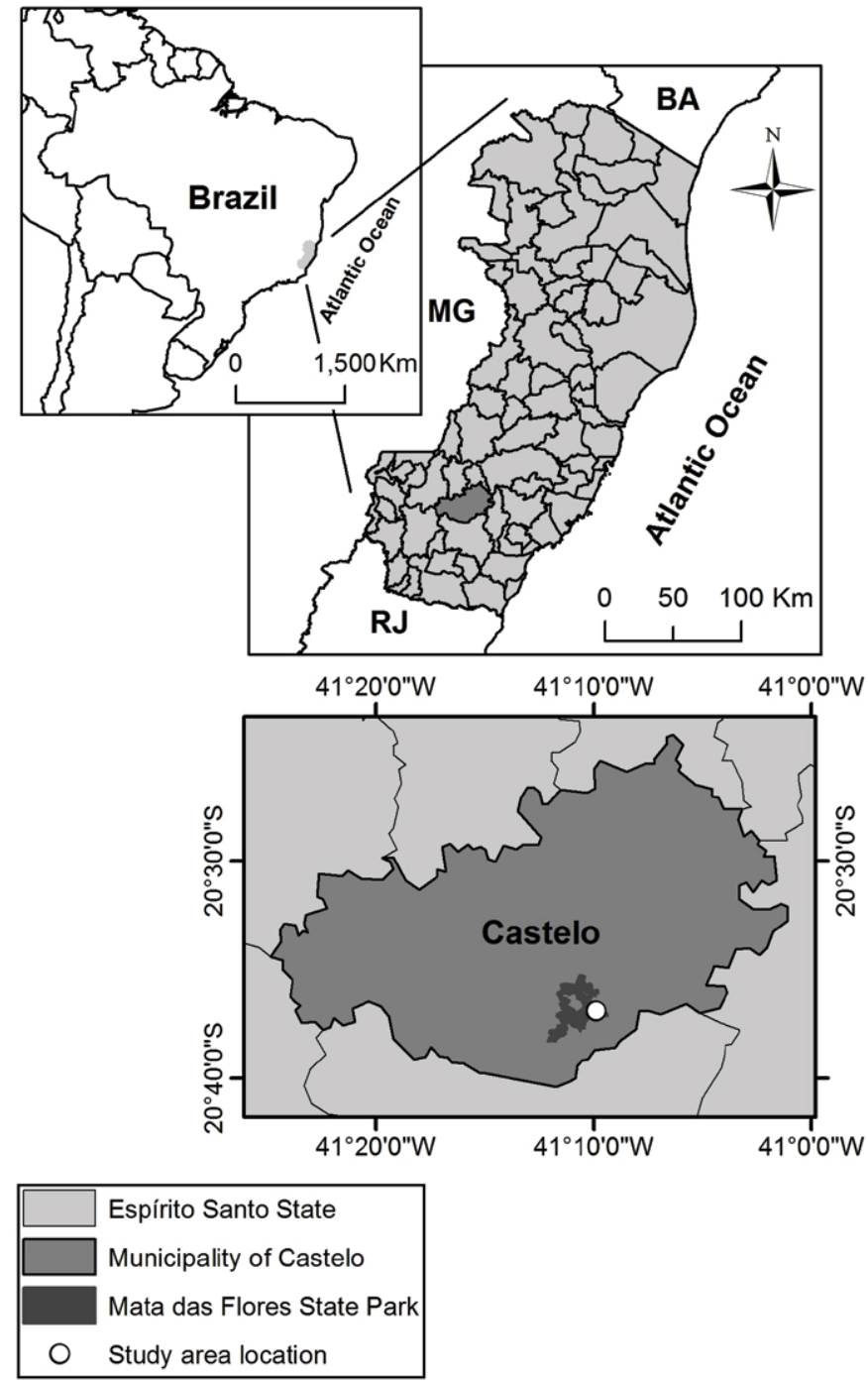

Figure 1. Location of the studied fragment. Top left, location of Espírito Santo state in Brazil. Top right, location of Castelo Municipality within Espírito Santo. Bottom, location of the fragment within Mata das Flores State Park (800 ha. of total area) in Castelo.

were collected and dried following usual procedures in plant collection (Peixoto and Maia 2013), and identified using specialized literature, consults to specialists (see Acknowledgements) and to the collections of RB, VIES (acronyms follow Thiers 2015), as well as the virtual herbarium REFLORA (http://reflora.jbrj.gov.br/ jabot/PrincipalUC/PrincipalUC.do). The samples were deposited at VIES, with duplicates sent to RB.

Family and genera were listed in alphabetical order following APG III (2009), including only indigenous species. Endemic species were marked with a circle $(\bullet)$ and new records for $\mathrm{ES}$ with a diamond $(\diamond)$. Identifications made by specialists based on non-fertile specimens were included in the list and marked with an asterisk $\left({ }^{*}\right)$, without designation of a collector number. The currently accepted names were verified in the List of Species of the Brazilian Flora (LSBF; http://floradobrasil.jbrj.gov. $\mathrm{br} /$ ). Conservation status was attributed following the 
Red Book of the Brazilian Flora (RBBF; Martinelli et al. 2013) and the Endangered Species Flora of the Espírito Santo State (ESFES; Fraga et al. 2007).

The LSBF and SL (CRIA, http://splink.cria.org.br/) websites were used as sources to assign a species as a new occurrence to ES. We considered that a species has a new occurrence in ES when it was not listed in LSBF, and a voucher specimen identified by a specialist was not located in herbaria collections using SL. The LSBF was also used to establish a comparison between the species richness of the families in MFSP and ES. The
SL was also used to check how many specimens for a particular species are included in herbaria collections. Both databases were consulted after the last updating of the LSBF (between 14 August and 17 August 2015).

\section{RESULTS}

The inventory resulted in 61 families, 159 genera and 239 species (Table 1). The families with the highest number of species were Rubiaceae (25 spp.), Piperaceae (19 spp.), Myrtaceae (13 spp.), Moraceae (10 spp.) and Fabaceae (9 spp.). These families comprised about $31 \%$ of all identified

Table 1. List of angiosperms from Mata das Flores State Park, ES, Brazil. $\triangle$ New records for ES $\bullet$ Endemic species of ES; ${ }^{*}$ Species identified based on non-fertile specimens. Red List (IUCN 2013) Status in Brazil: LC = least concern, VU = vulnerable, EN = endangered, CR = critically endangered. RBBF - Red Book of the Brazilian Flora; ESFES - Endangered Species of the Espírito Santo state Flora.

\begin{tabular}{|c|c|c|c|}
\hline Family/Species & $\begin{array}{l}\text { RBBF } \\
\text { list }\end{array}$ & $\begin{array}{l}\text { ESFES } \\
\text { list }\end{array}$ & Voucher \\
\hline \multicolumn{4}{|l|}{ Acanthaceae } \\
\hline Aphelandra blanchetiana (Nees) Hook. & & & T.T.Carrijo 1717 \\
\hline Aphelandra longiflora (Lindl.) Profice & & & T.T.Carrijo 1777 \\
\hline Aphelandra maximiliana (Nees) Benth. • & EN & EN & T.T.Carrijo 1689 \\
\hline Justicia genuflexa Nees \& Mart. & VU & VU & T.T.Carrijo 1712 \\
\hline Justicia parahyba P.L.R.Moraes & & & T.T.Carrijo 1876 \\
\hline Justicia wasshauseniana Profice & & VU & T.T.Carrijo 1851 \\
\hline Ruellia solitaria Vell. & & & T.T.Carrijo 2046 \\
\hline Schaueria lachnostachya Nees & & & T.T.Carrijo 1540 \\
\hline \multicolumn{4}{|l|}{ Achariaceae } \\
\hline Carpotroche brasiliensis (Raddi) A. Gray & & & T.T.Carrijo 1945 \\
\hline \multicolumn{4}{|l|}{ Amaranthaceae } \\
\hline Chamissoa acuminata Mart. $\downarrow$ & & & T.T.Carrijo 1891 \\
\hline Chamissoa altissima (Jacq.) Kunth & & & T.T.Carrijo 1656 \\
\hline \multicolumn{4}{|l|}{ Apocynaceae } \\
\hline Asclepias curassavica L. & & & T.T.Carrijo 2215 \\
\hline Oxypetalum banksii R.Br. ex Schult. & & & T.T.Carrijo 1705 \\
\hline Rauvolfia capixabae I.Koch \& Kin.-Gouv. & & & T.T.Carrijo 1568 \\
\hline Tabernaemontana hystrix Steud. & & & T.T.Carrijo 1796 \\
\hline \multicolumn{4}{|l|}{ Araceae } \\
\hline Monstera adansonii Schott & & & T.T.Carrijo 1757 \\
\hline Philodendron loefgrenii Engl. & & & T.T.Carrijo 1908 \\
\hline Syngonium vellozianum Schott & & & T.T.Carrijo 2160 \\
\hline \multicolumn{4}{|l|}{ Arecaceae } \\
\hline Astrocaryum aculeatissimum (Schott) Burret & & & T.T.Carrijo 1622 \\
\hline Desmoncus polyacanthos Mart. & & & T.T.Carrijo 1809 \\
\hline Geonoma elegans Mart. & & & T.T.Carrijo 1581 \\
\hline \multicolumn{4}{|l|}{ Aristolochiaceae } \\
\hline Aristolochia cymbifera Mart. \& Zucc. & & & J. Freitas 203 \\
\hline \multicolumn{4}{|l|}{ Asparagaceae } \\
\hline Herreria glaziovii Lecomte & & & T.T.Carrijo 2077 \\
\hline \multicolumn{4}{|l|}{ Asteraceae } \\
\hline Ageratum conyzoides L. & & & T.T.Carrijo 1813 \\
\hline Baccharis trinervis Pers. & & & T.T.Carrijo 1860 \\
\hline $\begin{array}{l}\text { Chromolaena maximilianii (Schrad. ex DC.) } \\
\text { R.M.King \& H.Rob. }\end{array}$ & & & T.T.Carrijo 1750 \\
\hline Cyathula prostrata Blume & & & T.T.Carrijo 1742 \\
\hline Emilia fosbergii Nicolson & & & T.T.Carrijo 1812 \\
\hline Piptocarpha cf. axillaris (Less.) Baker $\diamond$ & & & T.T.Carrijo 1797 \\
\hline Synedrella nodiflora (L.) Gaertn. & & & T.T.Carrijo 1814 \\
\hline Vernonanthura ferruginea (Less.) H.Rob. $\diamond$ & & & T.T.Carrijo 1803 \\
\hline \multicolumn{4}{|l|}{ Balanophoraceae } \\
\hline Langsdorffia hypogaea Mart. & & & T.T.Carrijo 1415 \\
\hline
\end{tabular}

\begin{tabular}{|c|c|c|c|}
\hline Family/Species & $\begin{array}{l}\text { RBBF } \\
\text { list }\end{array}$ & $\begin{array}{l}\text { ESFES } \\
\text { list }\end{array}$ & Voucher \\
\hline \multicolumn{4}{|l|}{ Begoniaceae } \\
\hline Begonia fischeri Schrank & & & T.T.Carrijo 1809 \\
\hline Begonia hirtella Link & & EN & T.T.Carrijo 1911 \\
\hline \multicolumn{4}{|l|}{ Bignoniaceae } \\
\hline Adenocalymma trifoliatum (Vell.) R.C.Laroche & & & T.T.Carrijo 1691 \\
\hline Bignonia aequinoctialis L. & & & T.T.Carrijo 1653 \\
\hline Bignonia campanulata Cham. & & & T.T.Carrijo 1571 \\
\hline Tanaecium selloi (Spreng.) L.G.Lohmann & & & T.T.Carrijo 1643 \\
\hline \multicolumn{4}{|l|}{ Boraginaceae } \\
\hline Cordia taguahyensis Vell. & & & T.T.Carrijo 2056 \\
\hline Heliotropium angiospermum Murray $\diamond$ & & & T.T.Carrijo 1584 \\
\hline Myriopus paniculatus (Cham.) Feuillet & & & T.T.Carrijo 1886 \\
\hline \multicolumn{4}{|l|}{ Bromeliaceae } \\
\hline Aechmea ramosa Mart. ex Schult. \& Schult.f. & & & T.T.Carrijo 2231 \\
\hline Billbergia cf. horrida Regel & & & T.T.Carrijo 1806 \\
\hline Tillandsia usneoides (L.) L. & & & T.T.Carrijo 1785 \\
\hline Tillandsia loliacea Mart. ex Schult. \& Schult.f. & & & T.T.Carrijo 1774 \\
\hline Vriesea carinata Wawra & & & T.T.Carrijo 1935 \\
\hline Vriesea ensiformis (Vell.) Beer & & & T.T.Carrijo 1701 \\
\hline \multicolumn{4}{|l|}{ Cactaceae } \\
\hline Pereskia aculeata Mill. & & & T.T.Carrijo 1719 \\
\hline \multicolumn{4}{|l|}{ Calophyllaceae } \\
\hline Calophyllum brasiliense Cambess. & & & T.T.Carrijo 1948 \\
\hline \multicolumn{4}{|l|}{ Cannabaceae } \\
\hline Trema micrantha (L.) Blume & & & T.T.Carrijo 1680 \\
\hline \multicolumn{4}{|l|}{ Celastraceae } \\
\hline Cheiloclinium cognatum (Miers) A.C.Sm. & & & T.T.Carrijo 1951 \\
\hline Hippocratea volubilis L. & & & T.T.Carrijo 1410 \\
\hline \multicolumn{4}{|l|}{ Commelinaceae } \\
\hline Commelina obliqua Vahl & & & T.T.Carrijo 1496 \\
\hline Dichorisandra incurva Mart. ex Schult.f. & & & T.T.Carrijo 1599 \\
\hline Dichorisandra thyrsiflora J.C.Mikan & & & T.T.Carrijo 1635 \\
\hline $\begin{array}{l}\text { Dichorisandra nutabilis Aona \& M.C.E.Amaral } \\
\text { - }\end{array}$ & & & T.T.Carrijo 2181 \\
\hline Tripogandra diuretica (Mart.) Handlos & & & T.T.Carrijo 2057 \\
\hline Tripogandra warmingiana (Seub.) Handlos & & & T.T.Carrijo 1805 \\
\hline Tradescantia zebrina Heynh. ex Bosse & & & T.T.Carrijo 1636 \\
\hline \multicolumn{4}{|l|}{ Costaceae } \\
\hline Costus spiralis (J.A.Christq.) Roscoe & & & T.T.Carrijo 1616 \\
\hline \multicolumn{4}{|l|}{ Cucurbitaceae } \\
\hline $\begin{array}{l}\text { Gurania bignoniaceae (Poepp. \& Endl.) } \\
\text { C.Jeffrey }\end{array}$ & & & T.T.Carrijo 2150 \\
\hline Wilbrandia verticillata (Vell.) Cogn. & & & T.T.Carrijo 1940 \\
\hline
\end{tabular}


Table 1. Continued.

\begin{tabular}{|c|c|c|c|}
\hline Family/Species & $\begin{array}{l}\text { RBBF } \\
\text { list }\end{array}$ & $\begin{array}{l}\text { ESFES } \\
\text { list }\end{array}$ & Voucher \\
\hline \multicolumn{4}{|l|}{ Cyperaceae } \\
\hline Cyperus surinamensis Rottb. & & & L.A.Silva 221 \\
\hline Pycreus unioloides (R.Br.) Urb. $\diamond$ & & & T.T.Carrijo 1609 \\
\hline \multicolumn{4}{|l|}{ Erythroxylaceae } \\
\hline Erythroxylum macrocalyx Mart. & & & T.T.Carrijo 1655 \\
\hline \multicolumn{4}{|l|}{ Euphorbiaceae } \\
\hline Acalypha cf. communis Müll. Arg. $\diamond$ & & & T.T.Carrijo 1605 \\
\hline Actinostemon concolor (Spreng.) Müll.Arg. & & & T.T.Carrijo 1895 \\
\hline Actinostemon verticillatus (Klotzsch) Baill. & & & T.T.Carrijo 1494 \\
\hline Mabea fistulifera Mart. & & & T.T.Carrijo 1417 \\
\hline Pachystroma longifolium (Ness) I.M. Johnst. & & & T.T.Carrijo 1787 \\
\hline Pausandra morisiana (Casar.) Radlk. & & & T.T.Carrijo 2164 \\
\hline Senefeldera verticillata (Vell.) Crozait & & & * \\
\hline \multicolumn{4}{|l|}{ Fabaceae } \\
\hline Aeschynomene americana $\mathrm{L}$. & & & T.T.Carrijo 1806 \\
\hline Bauhinia forficata Link & & & T.T.Carrijo 2088 \\
\hline Desmodium subsericeum Malme & & & T.T.Carrijo 1721 \\
\hline Inga hispida Schott ex Benth. & & & * \\
\hline Parapiptadenia pterosperma (Benth.) Brenan & & & * \\
\hline Peltophorum dubium (Spreng.) Taub. & & & T.T.Carrijo 1626 \\
\hline Piptadenia gonoacantha (Mart.) J.F.Macbr. & & & L.A.Silva 404 \\
\hline Senna affinis (Benth.) H.S.Irwin \& Barneby & & & T.T.Carrijo 2103 \\
\hline $\begin{array}{l}\text { Senegalia giganticarpa (G.P.Lewis) Seigler \& } \\
\text { Ebinger }\end{array}$ & & & T.T.Carrijo 1795 \\
\hline
\end{tabular}



\begin{tabular}{|c|c|c|c|}
\hline Family/Species & $\begin{array}{l}\text { RBBF } \\
\text { list }\end{array}$ & $\begin{array}{l}\text { ESFES } \\
\text { list }\end{array}$ & Voucher \\
\hline \multicolumn{4}{|l|}{ Meliaceae } \\
\hline Guarea guidonia (L.) Sleumer & & & T.T.Carrijo 1748 \\
\hline Trichillia hirta L. & & & T.T.Carrijo 1773 \\
\hline Trichilia pseudostipularis (A.Juss.) C.DC. & & & T.T.Carrijo 2153 \\
\hline \multicolumn{4}{|l|}{ Menispermaceae } \\
\hline Abuta convexa (Vell.) Diels & & & * \\
\hline $\begin{array}{l}\text { Chondrodendron platiphyllum (A.St.-Hil.) } \\
\text { Miers }\end{array}$ & & & T.T.Carrijo 1894 \\
\hline $\begin{array}{l}\text { Hyperbaena oblongifolia (Mart.) Chodat \& } \\
\text { Hassl. } \diamond\end{array}$ & & & T.T.Carrijo 1778 \\
\hline \multicolumn{4}{|l|}{ Monimiaceae } \\
\hline Mollinedia sphaerantha Perkins & & & T.T.Carrijo 2155 \\
\hline Mollinedia widgrenii A.DC. $\diamond$ & & & T.T.Carrijo 1960 \\
\hline \multicolumn{4}{|l|}{ Moraceae } \\
\hline Brosimum guianense (Aubl.) Huber & & & M.C. Santos 03 \\
\hline Clarisia ilicifolia (Spreng.) Lanj. \& Rossberg & & & T.T.Carrijo 1572 \\
\hline Dorstenia arifolia Lam. & & & T.T.Carrijo 1516 \\
\hline Dorstenia elata Hook. & & & T.T.Carrijo 1618 \\
\hline Dorstenia bonijesu Carauta \& C. Valente & & Vu & T.T.Carrijo 1939 \\
\hline Dorstenia hirta Desv. & & & T.T.Carrijo 1556 \\
\hline Ficus eximia Schott. & & & M.C. Santos 01 \\
\hline Ficus obtusiuscula (Miq.) Miq. & & & T.T.Carrijo 2008 \\
\hline Naucleopsis oblongifolia (Kuhlm.) Carauta & & & - \\
\hline Sorocea hillari Gaudich. & & & T.T.Carrijo 1768 \\
\hline \multicolumn{4}{|l|}{ Myristicaceae } \\
\hline Virola bicuhyba (Schott ex Spreng.) Warb. & & & * \\
\hline
\end{tabular}

Eugenia bahiensis DC.

LC

T.T.Carrijo 2059

Eugenia candolleana DC.

Eugenia excelsa O.Berg

Eugenia itapemirimensis Cambess.

Eugenia melanogyna (D.Legrand) Sobral

Eugenia neogracilis Mazine \& Sobral

Eugenia pisiformis Cambess. $\quad$ LC $\quad$ T.T.Carrijo 1492

Eugenia prasina O.Berg $\quad$ LC

Marlierea silvatica (O.Berg) Kiaersk.•

Myrcia follii G.M.Barroso \& Peixoto •

Myrcia guianensis (Aubl.) DC.

Myrcia limae G.M.Barroso \& Peixoto • EN VU T.T.Carrijo 2218

Myrcia splendes (Sw.) DC. T.T.Carrijo 1686

\section{Nyctaginaceae}

Bougainvillea spectabilis Willd.

T.T.Carrijo 1798

Guapira opposita (Vell.) Reitz

Leucaster caniflorus (Mart.) Choisy

Ramisia brasiliensis Oliv.

T.T.Carrijo 1560

Ochnacea

Ouratea parviflora (A.DC.) Baill.

T.T.Carrijo 2010

\section{Orchidaceae}

Oeceoclades maculata (Lindl.) Lindl.

T.T.Carrijo 1498

Rodriguezia venusta Rchb.f.

Sacoila lanceolata (Aubl.) Garay

T.T.Carrijo 1663

T.T.Carrijo 1850

T.T.Carrijo 1411

\section{Oxalidaceae}

Oxalis bela-vitoriae Lourteig $\diamond$

T.T.Carrijo 1559

Oxalis cytisoides Mart. ex Zucc.

CR

VU T.T.Carrijo 1720

Passiflora kermesiana Link \& Otto

T.T.Carrijo 1727

\section{Phytolacaceae}

Rivina humilis L.

T.T.Carrijo 1799 
Table 1. Continued.

\begin{tabular}{|c|c|c|c|}
\hline Family/Species & $\begin{array}{l}\text { RBBF } \\
\text { list }\end{array}$ & $\begin{array}{l}\text { ESFES } \\
\text { list }\end{array}$ & Voucher \\
\hline \multicolumn{4}{|l|}{ Picramniaceae } \\
\hline Picramnia parvifolia Engl. $\diamond$ & & & T.T.Carrijo 1500 \\
\hline \multicolumn{4}{|l|}{ Piperaceae } \\
\hline Piper aduncum $\mathrm{L}$. & & & T.T.Carrijo 1583 \\
\hline Piper amalago L. & & & T.T.Carrijo 1628 \\
\hline Piper anisum (Spreng.) Angely & & & T.T.Carrijo 1654 \\
\hline Piper arboreum Aubl. & & & J.A.Christ 26 \\
\hline Piper bowiei Yunck. & & & J.A.Christ 93 \\
\hline Piper caldense C.DC. & & & T.T.Carrijo 1645 \\
\hline Piper cernuum Vell. & & & J.A.Christ 92 \\
\hline Piper dilatatum Rich & & & J.A.Christ 74 \\
\hline Piper gaudichaudianum Kunth & & & T.T.Carrijo 1612 \\
\hline Piper glabratum Kunth & & & T.T.Carrijo 1585 \\
\hline Piper hoffmannseggianum Roem. \& Schult & & & T.T.Carrijo 1543 \\
\hline Piper macedoi Yunck. & & & T.T.Carrijo 1586 \\
\hline Piper miquelianum C.DC. & & & T.T.Carrijo 1802 \\
\hline Piper permucronatum Yunck. & & & T.T.Carrijo 1668 \\
\hline Piper piliovarium Yunck. $\diamond$ & & & T.T.Carrijo 1632 \\
\hline Piper pubisubmarginalum Yunck. & & & T.T.Carrijo 1688 \\
\hline Piper tuberculatum Jacq. & & & T.T.Carrijo 1666 \\
\hline Piper umbellatum L. & & & J.A.Christ 99 \\
\hline Piper vicosanum Yunck. & & EN & J.A.Christ 76 \\
\hline \multicolumn{4}{|l|}{ Plumbaginaceae } \\
\hline Plumbago scandens L. & & & T.T.Carrijo 1740 \\
\hline \multicolumn{4}{|l|}{ Poaceae } \\
\hline Cenchrus purpureus (Schumach.) Morrone & & & T.T.Carrijo 1747 \\
\hline \multicolumn{4}{|l|}{ Primulaceae } \\
\hline Clavija caloneura Mart. & & & T.T.Carrijo 1710 \\
\hline Stylogyne warmingii Mez. & & & T.T.Carrijo 1534 \\
\hline \multicolumn{4}{|l|}{ Rubiaceae } \\
\hline Alseis floribunda Schott & & & F.T.Leite 42 \\
\hline Amaioua guianensis Aubl. & & & F.T.Leite 57 \\
\hline $\begin{array}{l}\text { Cordiera myrciifolia (K. Schum.) C. H. Perss. } \\
\text { \& Delprete }\end{array}$ & & & F.T.Leite 35 \\
\hline Carapichea ipecacuanha (Brot.) L. Andersson & VU & & F.T.Leite 43 \\
\hline Genipa americana L. & & & F.T.Leite 111 \\
\hline Hamelia patens Jacq. & & & T.T.Carrijo 1669 \\
\hline Faramea campanella Müll.Arg. & & & T.T.Carrijo 1684 \\
\hline Faramea coerulea (Nees \& Mart.) DC. & & & T.T.Carrijo 1677 \\
\hline Faramea involucellata Müll.Arg. & & & T.T.Carrijo 1957 \\
\hline Faramea martiana Müll.Arg. & & & T.T.Carrijo 1525 \\
\hline Faramea multiflora A.Rich. ex DC. & & & T.T.Carrijo 1763 \\
\hline Faramea sellowiana Benth. & & & T.T.Carrijo 1704 \\
\hline Geophila repens (L.) I.M.Johnst. & & & F.T.Leite 49 \\
\hline $\begin{array}{l}\text { Margaritopsis cephalantha (Müll.Arg.) } \\
\text { C.M.Taylor }\end{array}$ & & & F.T.Leite 69 \\
\hline Margaritopsis chaenotricha (DC.) C.M. Taylor & & & F.T.Leite 62 \\
\hline Psychotria carthagenensis Jacq. & & & F.T.Leite 96 \\
\hline Psychotria deflexa DC. & & & F.T.Leite 80 \\
\hline Psychotria stellaris Müll.Arg. & & & T.T.Carrijo 1548 \\
\hline Psychotria rhytidocarpa Müll. Arg. & & & T.T.Carrijo 1547 \\
\hline Psychotria minutiflora Müll.Arg. & & & T.T.Carrijo 1936 \\
\hline $\begin{array}{l}\text { Rudgea coronata (Vell.) Müll. Arg. subsp. } \\
\text { coronata }\end{array}$ & & & T.T.Carrijo 1607 \\
\hline Rudgea coronata subsp. ochroleuca (Müll.Arg.) & & & T.T.Carrijo 1514 \\
\hline $\begin{array}{l}\text { Rudgea coronata subsp. saint-hilaire } \\
\text { (Standl.) Zappi } \bullet\end{array}$ & CR & $\mathrm{CR}$ & T.T.Carrijo 1484 \\
\hline
\end{tabular}

\begin{tabular}{llll}
\hline Family/Species & $\begin{array}{l}\text { RBBF } \\
\text { list }\end{array}$ & $\begin{array}{l}\text { ESFES } \\
\text { list }\end{array}$ & Voucher \\
\hline Rudgea reflexa Zappi & & EN & T.T.Carrijo 1579 \\
Sabicea villosa Willd. Ex. Schult. & & & F.T.Leite 85 \\
\hline Rutaceae & & \\
Erythrochiton brasiliensis Ness \& Mart. & & T.T.Carrijo 1518 \\
Almeidea rubra A.St.-Hil. & & T.T.Carrijo 1575 \\
Rauia resinosa Nees \& Mart. & & T.T.Carrijo 1580 \\
Conchocarpus macrophyllus J.C.Mikan & & T.T.Carrijo 1945 \\
Esenbeckia pilocarpoides Kunth & T.T.Carrijo 1767 \\
Pilocarpus giganteus Engl. & & T.T.Carrijo 1761 \\
Neoraputia alba (Nees \& Mart.) Emmerich & & T.T.Carrijo 1952 \\
ex Kallunki & &
\end{tabular}

Salicaceae

Casearia espiritosantensis R. Marquete \&

Mansano•

Casearia souzae R. Marquete \& Mansano

Casearia sylvestris Sw.

T.T.Carrijo 1715

Prockia crucis P. Browne ex L.

\section{Sapindaceae}

Paullinia meliifolia Juss. $\diamond$

T.T.Carrijo 1909

Cardiospermum grandiflorum Sw.

Allophylus melanophloeus Radlk. $\diamond$

T.T.Carrijo 2196

Serjania communis Cambess.

T.T.Carrijo 2212

T.T.Carrijo 1476

T.T.Carrijo 1735

Matayba guianensis Aubl.

T.T.Carrijo 1976

\section{Sapotaceae}

Chrysophyllum gonocarpum (Mart. \& Eichler

L.A.Silva 403

ex Miq.) Engl.

Chrysophyllum lucentifolium Cronquist

Ecclinusa guianensis Eyma

*

Sarcaulus brasiliensis (A.DC.) Eyma

Pouteria bangii (Rusby) T.D.Penn.

Pouteria durlandii (Standl.) Baehni

Pouteria guianensis Aubl.

T.T.Carrijo 1502

Pouteria filipes Eyma

\section{Simaroubaceae}

Picramnia crenata (Vell.) Engl. $\diamond$

M.Zanetti 01

\section{Smilacaceae}

Smilax spicata Vell.

T.T.Carrijo 1629

\section{Solanacea}

Physalis angulata $\mathrm{L}$.

T.T.Carrijo 2213

Solanum aculeatissimum Jacq. $\diamond$

Solanum campaniforme Roem. \& Schut.

Solanum lacteum Vell.

Solanum sublentum Hiern

T.T.Carrijo 1792

T.T.Carrijo 2063

T.T.Carrijo 1801

Stemonuraceae

Discophora guianensis Miers

T.T.Carrijo 2047

\section{Urticaceae}

Laportea aestuans (L.) Chew

T.T.Carrijo 1569

Pilea hyalina Fenzl

Pourouma guianensis Aubl.

Urera baccifera (L.) Gaudich. ex Wedd.

T.T.Carrijo 1728

T.T.Carrijo 1808

T.T.Carrijo 2168

\section{Verbenaceae}

Lantana camara L.

T.T.Carrijo 1758

Stachytarpheta jamaicensis (L.) Vahl

T.T.Carrijo 1946

\section{Violaceae}

Noisettia orchidiflora (Rudge) Ging
T.T.Carrijo 2055

T.T.Carrijo 1613 
species. Twenty-two families were represented by only one species each, totalizing about $9 \%$ of all identified species. Considering the criteria previously explained, 21 species are new records for ES, eight are endemic to this state, and 20 are listed as vulnerable, endangered or critically endangered in the RBBF (Martinelli et al. 2013) or in the ESFES (Fraga et al. 2007).

Amongst Rubiaceae, Rudgea coronata subsp. sainthilaire (Standl.) Zappi (Figure 2a, b) is endemic of the $E S$, and is indicated as critically endangered $(C R)$ in both
RBBF and ESFES lists. This taxon is relatively common in the park, occurring strictly within the forest, and their individuals aggregate in relatively small populations. The occurrence of Rudgea coronata subsp. saint-hilaire in the ES was attested by only two collections, both from the Linhares municipality, northern Espírito Santo. Thus, the collections of MFSP expanded the geographic distribution of this species to the southwards of the state. Another species of the same genus, R. reflexa Zappi (Figure 2c) is considered endangered (EN) in RBBF, but
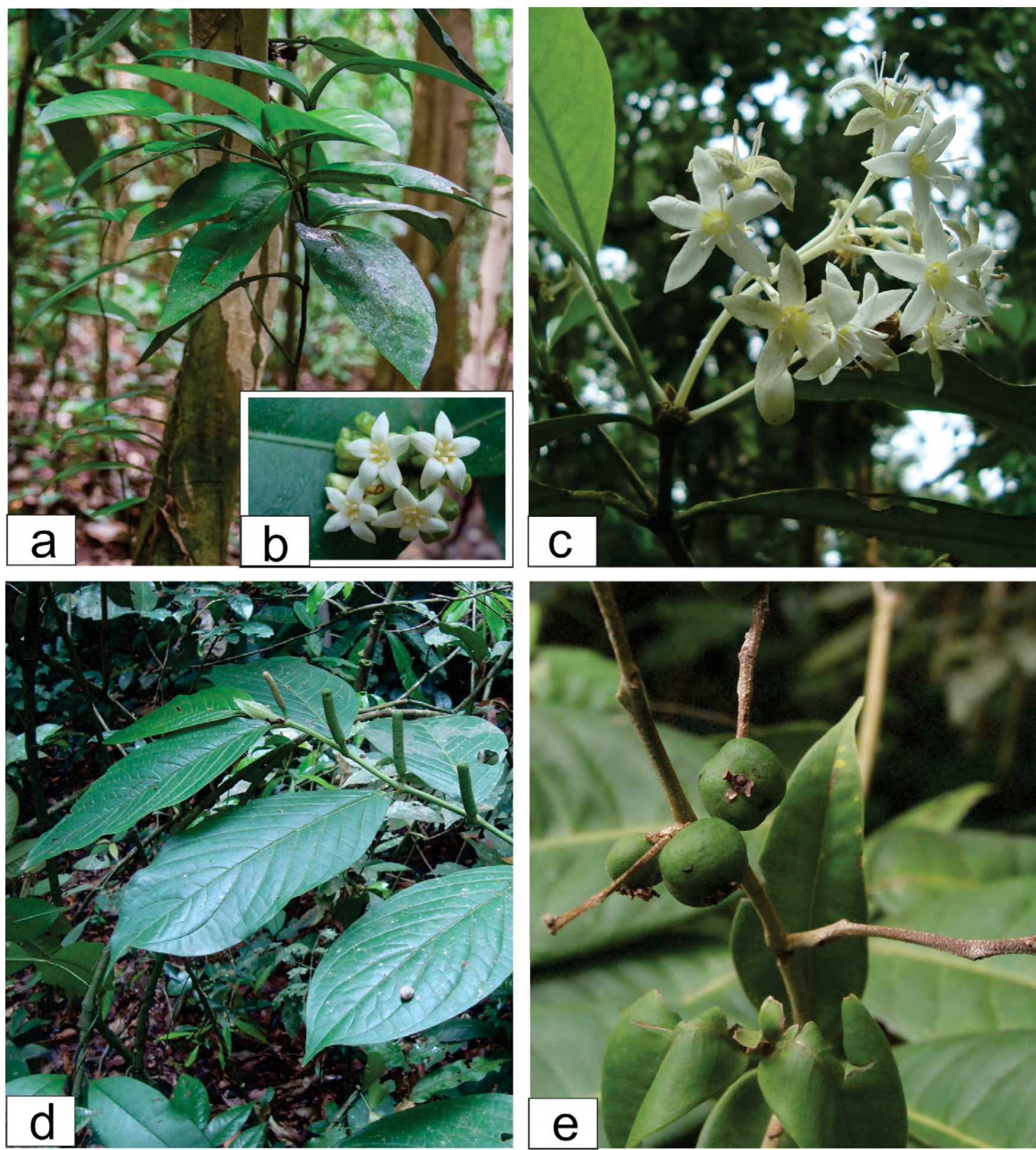

Figure 2. Some of the plant species found in the Mata das Flores State Park. A-B. Rudgea coronata ssp. saint-hillaire (habit and inflorescence); C. Rudgea reflexa (inflorescence); D. Piper miquelianum (inflorescence); E. Myrcia limae (immature fruits). 
is not endemic to the Espírito Santo, also occurring in Bahia state. Rudgea reflexa and $R$. coronata subsp. sainthilaire are very common understory species in the MFSP, being exclusively found in this layer of the forest.

None of the Piperaceae species that occur in the MFSP is endemic to Espírito Santo, and Piper piliovarium Yunck is a new occurrence for this state. Individuals of this species occur strictly within the forest and show an aggregated distribution. Piper vicosanum Yunck. (Figure 2d) is the only species categorized under some threat criteria. The species is considered endangered (EN) in the ESFES list. In the MFSP, isolated individuals occur both inside the forest and in roadsides.

Amongst the three species of Myrtaceae that are endemic of ES and occur in the MFSP, two are categorized in some of the IUCN (2013) threat category. Myrcia limae Barroso and Peixoto (Figure 2e) is also an endemic species of the ES, categorized as endangered (EN) by RBBF and vulnerable (VU) by the ESFES lists. This species was previously known by few collections from four municipalities (Conceição da Barra, Nova Venécia, Linhares and Santa Teresa). The distribution of M. limae is therefore expanded in Espírito Santo. Moreover, the specimens collected in the MFSP also add to the knowledge about the morphology of the fruits of this species, which were not described at the species' protologue. Another species of note in the same genus is Myrcia follii Barroso \& Peixoto. This species is indicated as critically endangered $(\mathrm{CR})$ in the RBBF and vulnerable (VU) in the ESFES, and was previously known for 20 samples gathered in three municipalities of Espírito Santo.

The genus Dorstenia (Moraceae) comprises four species in MFSP, which apparently occur in different habitats. Topography and light seem to explain the differences in the spatial organization of the different species in the park. Populations of D. arifolia (Figure $3 a, b)$ are always ombrophilous and occur in aggregated patches, preferentially in hill slopes. D. bonijesu (Figure 3c) does not show an aggregate pattern of organization. Its isolated individuals occur in both shaded areas of hilltops, and open areas of roadsides. This species was classified as vulnerable (VU) in the ESFES. However, in the MFSP this species is quite common, occurring even in disturbed areas at roadsides. Individuals show a low size variation. Finally, D. elata (Figure 3d, e) occurs exclusively in shaded forest interiors instead. At last, populations of $D$. hirta (Figure $3 f, g$ ) were found in shaded areas and below canopy gaps. In these sites, observed individuals varied from $20 \mathrm{~cm}$ to $1 \mathrm{~m}$ height, respectively.

Even though Rutaceae presented low species richness in the studied site, Almeidea rubra A.St.-Hil. is the most commonly found understory species, as well as Clavija caloneura Mart. ex Miq. (Primulaceae). The individuals of A. rubra in MFSP show an aggregated distribution in space, occurring from valleys to hilltops. Individuals of
C. caloneura, on the contrary, were commonly observed at the valleys, and apparently are not distributed in aggregate populations.

\section{DISCUSSION}

Our results show that the conservation value of small sized fragments (ca. 150 ha) can be quite high, considering their overall species richness and number of endangered species they can hold. It is noteworthy that such small remnants can comprise about $12 \%$ of what remains in forest cover from this biome (Ribeiro et al. 2009). Our results also highlight the need for time consuming, intensive, floristic inventories in order to encompass the flowering time of most plant species occurring in a site. Because snapshot inventories may not detect several plant species with short flowering periods, we concentrated the sampling effort within this 144 ha remnant for a relatively long period (two years), with a high visiting frequency (weekly).

The overall pattern of species richness, especially within the families Rubiaceae, Piperaceae, and Myrtaceae, is that expected for Lowland Dense Ombrophilous Forests (Carvalho 2006) and other physiognomies of the Atlantic Forest (Amorim et al. 2009; Carvalho et al. 1996; Carvalho et al. 2000). Floristic surveys in different vegetation types of Espírito Santo confirm the high richness of Myrtaceae and Rubiaceae in this state (Assis et al. 2004; Jesus and Rolim 2005; Rolim et al. 2006; Saiter et al. 2011). However, the high species richness of Piperaceae was an unexpected result, considering that Myrtaceae is the richest angiosperm family of the Brazilian flora (Forzza et al 2010), and is highly representative in the Atlantic Forest (Mori et al. 1983; Oliveira-Filho and Fontes 2000). A possible explanation for this result is the fact that the increased heterogeneity created by trails and roads, and their related edge effects, seems to favor species of Piperaceae more than species of Myrtaceae. The open areas within the MFSP are represented by roads that cross the fragment and by trails inside the forest reportedly created by motocross practice in the past. Piper species usually occurs near roadsides and in trails inside the forest. The fruit dispersal made by bats is one possible reason to explain this spatial occupation (Thiers and Kalko 2004). These animals predominantly forage in open areas (Coffin 2007), leading seeds to fall during the flight.

The results found in MFSP reveal the importance of small forest fragments in conserving endemic species known for a small number of populations. Many other collection gaps in southeast Brazil deserve future floristic inventories, especially in the southern portion of Espírito Santo state. Field studies on small sized remnants within areas with collection gaps are essential to improve herbaria collections and biodiversity databases of the Atlantic Forest. 



Figure 3. Dorstenia species from Mata das Flores State Park. A-B. D. arifolia (habit and inflorescence); C. D. bonijesu (habit); D-E. D. elata (habit and inflorescence); F-G. D. hirta (habit and inflorescence). 


\section{ACKNOWLEDGEMENTS}

We thank FAPES (Espírito Santo Research Agency) and $\mathrm{CNPq}$ (Brazilian Research Agency) for funding (research grant number 59152982/2012); FAPES, CNPq and Capes for fellowships to M.L. Garbin; CAPES (Brazilian Graduate Training Agency) for graduate fellowship to A.C. Tuler; FAPES for graduate fellowships for K.G.G. Martins, J.A. Christ, and J. Luber; CNPq for undergraduate fellowships to F. Torres, R.K. Hollunder, and J. Luber; Centro de Ciências Agrárias, Universidade Federal do Espírito Santo for logistical support. We also thank the experts Anderson Alves Araújo (Sapotaceae), Elsie Franklin Guimarães (Piperaceae), Elton John de Lírio (Monimiaceae), Haroldo Cavalcante de Lima (Fabaceae), Leandro A. Silva (Fabaceae), Leandro Assis (Lauraceae), Leandro Cardoso Pederneiras (Moraceae), Marli Pires Morin (Fabaceae), Michel Ribeiro (Lecythidaceae), Ronaldo Marquete (Salicaceae) and Valquíria Dutra (Fabaceae) for plant identification. We are grateful to the IEMA (Espírito Santo environmental agency) staff in Mata das Flores State Park, for field support and collection licenses, and to the curators of VIES herbarium, for the agility in the inclusion of specimens in the collection. We also thank Joaquim Gasparini dos Santos, for photographs.

\section{LITERATURE CITED}

Amorim, A.M., J.G. Jardim, M.M.M. Lopes, P. Fiaschi, P.A.X. Borges, R. Perdiz and W.W. Thomas. 2009. Angiospermas em remanescentes de Floresta Montana no Sul da Bahia, Brasil. Biota Neotropica 9(3): 313-348. doi: 10.1590/S1676-06032009000300028

APG (Angiosperm Phylogeny Group). 2009. An update of the Angiosperm Phylogeny Group classification for the orders and families of flowering plants: APG III. Botanical Journal of the Linnean Society 161(2): 105-121. doi: 10.1111/j.10958339.2009.00996.x

Assis, A.M., L.D. Thomaz and O.J. Pereira. 2004. Florística de um trecho de floresta de restinga no município de Guarapari, Espírito Santo, Brasil. Acta Botanica Brasilica 18(1): 191-201. doi: 10.1590/S0102-33062004000100016

Baitello, J.B. 2001. Novas espécies de Lauraceae para a flora brasileira. Acta Botanica Brasilica 15(3): 445-450. doi: 10.159o/ So102-33062001000300012

Bini, L.M., J.A.F. Diniz-Filho, T.F.L.V.B. Rangel, R.P. Bastos and M.P. Pinto. 2006. Challenging Wallacean and Linnean shortfalls: knowledge gradients and conservation planning in a biodiversity hotspot. Diversity and Distributions 12(5): 475-482. doi: 10.1111/j.1366-9516.2006.00286.x

Brotto, M.L. and J.B. Baitello. 2012. Uma espécie nova de Lauraceae da Floresta Atlântica do Brasil. Rodriguésia 63(3): 579-585. http://rodriguesia-seer.jbrj.gov.br/index.php/rodriguesia/article/ view/391/203

Carvalho, D.A., A.T. Oliveira-Filho and E.A. Vilela. 1996. Flora arbustivo-arbórea de mata ripária do médio Rio Grande (Conquista, Estado de Minas Gerais). Cerne 2(2): 48-68. http://www.dcf.ufla. br/cerne/artigos/16-02-20093334V2_n2_artigo\%2004.pdf

Carvalho, D.A., A.T. Oliveira-Filho, E.A. Vilela and N. Curi. 2000. Florística e estrutura da vegetação arbórea semidecidual às margens do reservatório da usina hidrelétrica dona Rita (Itambé do Mato Dentro, MG). Acta Botanica Brasilica 14(1): 37-55. doi: 10.1590/S0102-33062000000100005
Carvalho, F.A., M.A. Nascimento and J.M. A.Braga. 2006. Composição e riqueza florística do componente arbóreo da Floresta Atlântica submontana na região de Imbaú, Município de Silva Jardim, RJ. Acta Botanica Brasilica 20(3): 727-740. doi: 10.1590/So10233062006000300022

Coelho, M.A.N. 2010. Espécies novas de Anthurium e Philodendron (Araceae) do sudeste brasileiro. Boletim do Museu de Biologia Mello Leitão 28: 21-4o. http://www.museudebiologiamelloleitao. gov.br/boletim/arquivos/28/Artigo-2_Coelho_28.pdf

Coffin, A.W. 2007. From road kill to road ecology: A review of the ecological effects of roads. Journal of Transport Geography 15(5): 396-406. doi:10.1016/j.jtrangeo.2006.11.006

CRIA (Centro de Referência em Informação Ambiental). 2014. SpeciesLink. Accessed at http://www.splink.org.br, 25 December 2014.

Deble, L.P. 2005. Novas espécies de Achyrocline (Less.) DC. (Asteraceae) para o sul do Brasil. Balduinia 3: 5-9. http://cascavel.ufsm. br/revistas/ojs-2.2.2/index.php/balduinia/article/view/14011

Deble, L.P. 2006. Um novo nome e duas novas combinações na Tribo Gnaphalieae (Asteraceae). Balduinia 6: 28-29. http:// cascavel.ufsm.br/revistas/ojs-2.2.2/index.php/balduinia/article/ view/14029

Forzza, R.C. (org.) et al. 2010. Catálogo de plantas e fungos. Rio de Janeiro: Andrea Jacobsson, Instituto de Pesquisas Jardim Botânico, v. 2. 828 pp. http://www.jbrj.gov.br/sites/all/themes/ corporateclean/content/publicacoes/plantas_fungos_vol2.pdf

Fraga, C.N., M. Simonelli and H.Q.B. Fernandes. 2007. Metodologia utilizada na elaboração da lista da flora ameaçada de extinção no Espírito Santo; pp. 59-72, in: C. N. Fraga and M. Simonelli. Espécies da flora ameaçadas de extinção no estado Espírito Santo. Vitória: IPEMA

Grand, J., M.O. Cummings, T.G. Rebelo, T.H. Ricketts and M.C. Neel. 2007. Biased data reduce efficiency and effectiveness of conservation reserve networks. Ecology Letters 10(5): 364-374. doi: 10.1111/j.1461-0248.2007.01025.x

IEMA (Instituto Estadual de Meio Ambiente e Recursos Hídricos). 2012. Parque Estadual de Mata das Flores. Accessed at http:// www.meioambiente.es.gov.br/, 15 May 2012.

IUCN (International Union for the Conservation of Nature). 2013. The IUCN Red List of threatened species. Version 2014.3. Accessed at http://www.iucnredlist.org, 25 December 2014.

Jesus, R.M. and S.G. Rolim. 2005. Fitossociologia da Mata Atlântica de Tabuleiro. Boletim Técnico da Sociedade de Investigações Florestais 19: 1-149.

Joly, C.A., J.P. Metzger and M. Tabarelli. 2014. Experiences from the Brazilian Atlantic Forest: ecological findings and conservation initiatives. New Phytologist 204(3): 459-473. doi: 10.1111/ nph.12989

List of Species of the Brazilian Flora. [2014]. Rio de Janeiro Botanical Garden. Accessed at http://floradobrasil.jbrj.gov.br/, 25 December 2014.

Margules, C.R. and R.L. Pressey. 2000. Systematic conservation planning. Nature 405: 243-253. doi: 10.1038/35012251

Martinelli, G and M.A. Moraes. 2013. Livro Vermelho da Flora do Brasil. Rio de Janeiro: Andrea Jacobsson: Instituto de Pesquisas Jardim Botânico. 1100 pp. http://cncflora.jbrj.gov.br/arquivos/ arquivos/pdfs/LivroVermelho.pdf

Martinelli, G., A.S.M. Valente, D. Maurenza, C. Kutschenko, D.M. Judice, D.S. Silva, E.P. Fernandez, E.M. Martins, F.S.M. Barros, J.C. Sfair, L.A.F. Santos-Filho, M.B. De Abreu, M.A. Moraes, N.P. Monteiro, P.V. Pietro, R.A. Fernandes, R.L.O. Hering, T. Messina and T.S.A. Penedo. 2013. Avaliações de risco de extinção de espécies da flora brasileira; pp. 6o-102, in: G. Martinnelli and M.A. Moraes (orgs.). Livro Vermelho da Flora do Brasil. Rio de Janeiro: Andrea Jacobsson, Instituto de Pesquisas Jardim Botânico. http:// cncflora.jbrj.gov.br/arquivos/arquivos/pdfs/Livro Vermelho.pdf 
Metzger, J.P. 2000. Tree functional group richness and landscape structure in a Brazilian tropical fragmented landscape. Ecological Applications 10(4): 1147-1161. doi: 10.1890/1051-0761 (2000)010[1147:tfgral]2.0.co;2

Mori, S.A., B.M. Boom, A.M. Carvalino and T.S. Santos. 1983. Ecological importance of Myrtaceae in an eastern Brazilian Wet Forest. Biotropica 15(1): 68-70. doi: 10.2307/2388002

Mori, S.A., B.M. Boom and G.T. Prance. 1981. Distribution patterns and conservation of eastern Brazilian coastal forest tree species. Brittonia 33(2): 233-245. doi: 10.2307/2806330

Murray-Smith, C., N.A. Brummitt, A.T. Oliveira-Filho, S. Bachman, J. Moat, E.M.N. Lughadha and E J. Lucas. 2008. Plant diversity hotspots in the Atlantic Costal Forests of Brazil. Conservation Biology 23(1): 151-163. doi: 10.1111/j.1523-1739.2008.01075.x

Oliveira, A.S. and L.P. Deble 2006. Duas novas espécies Sul-Brasileiras de Baccharis L. (Asteraceae-Astereae). Balduinia 9: 4-9. http:// cascavel.ufsm.br/revistas/ojs-2.2.2/index.php/balduinia/article/ view/14033

Oliveira-Filho, A.T. and M.A.L. Fontes. 2000. Patterns of floristic differentiation among Atlantic forests in Southeastern Brazil and the influence of climate. Biotropica 32(4b):793-810. doi: 10.1111/j.1744-7429.200o.tboo619.x

Oliveira-Filho, A.T., E. Tameirão-Neto, W.A.C. Carvalho, M. Werneck, A.E. Brima, C.V. Vidal, S.C. Rezende and J.A.A. Pereira. 2005 Análise florística do compartimento arbóreo de áreas de Floresta Atlântica sensu lato na região das Bacias do Leste (Bahia, Minas Gerais, Espírito Santo e Rio de Janeiro). Rodriguésia 56(87): 185-235. http://rodriguesia.jbrj.gov.br/FASCICULOS/ rodrig56_87/15Ary.pdf

Peixoto, A.L. 1992. Vegetação da Costa Atlântica; pp. 33-42, in: S. Monteiro and L. Kaz (coords.) Floresta Atlântica. Rio de Janeiro: Livroarte Editora.

Peixoto, A.L., and L.C. Maia 2013. Manual de Procedimentos para Herbários. INCT-Herbário virtual para a Flora e os Fungos. Recife: Editora Universitária UFPE. 97 pp. http://inct.florabrasil. net/wp-content/uploads/2013/11/Manual_Herbario.pdf

Pimm, S.L., C.N. Jenkins, R. Abell, T.M. Brooks, J.L. Gittleman, L.N. Joppa, P.H. Raven, C.M. Roberts and J. O. Sexton 2014. The biodiversity of species and their rates of extinction, distribution, and protection. Science 344 (6187): 1246752. doi: 10.1126/ science.1246752

Reflora - Herbário Virtual. [2014]. Jardim Botânico do Rio de Janeiro. Accessed at http://reflora.jbrj.gov.br/jabot/herbarioVirtual/, 25 December 2014.

Ribeiro, M.C., J.P. Metzger, A.C. Martensen, F.J. Ponzoni and M.M. Hirota. 2009. The Brazilian Atlantic Forest: How much is left, and how is the remaining forest distributed? Implications for conservation. Biological Conservation 142(6): 1141-1153. doi: 10.1016/j.biocon.2009.02.021

Rolim, S.G., N.M. Ivanauskas, R.R. Rodrigues, M.T. Nascimento, J.M.L. Gomes, D.A. Folli and H.T.Z. Couto. 2006. Composição Florística do estrato arbóreo da Floresta Estacional Semidecidual na Planície Aluvial do rio Doce, Linhares, ES, Brasil. Acta Botanica Brasilica 20(3):549-561. doi: 10.1590/So102-33062006000300005 Saiter, F.Z., F.A.G. Guilherme, L.D. Thomaz and T. Wendt. 2011. Tree changes in a mature rainforest with high diversity and endemism on the Brazilian coast. Biodiversity and Conservation 20(9): 1921-1949. doi: 10.1007/s10531-011-0067-3

Santos, K. and L.S. Kinoshita. 2003. Flora arbustivo-arbórea do fragmento de floresta estacional semidecidual do Ribeirão Cachoeira, município de Campinas, SP. Acta Botanica Brasilica 17(3): 325-341. doi: 10.1590/So102-33062003000300001

Stehmann, J.R., R.C. Forzza, A. Salino, M. Sobral, D.P. Costa and L. Kamino. 2009. Plantas da Floresta Atlântica. Rio de Janeiro: Jardim Botânico do Rio de Janeiro. 516 pp.

Thiers, B. [2015]. Index Herbariorum: a global directory of public herbaria and associated staff. New York Botanical Garden's Virtual Herbarium. Accessed at http://sweetgum.nybg.org/ih, 25 December 2014.

Thies, W. and E.K.V. Kalko. 2004. Phenology of Neotropical pepper plants (Piperaceae) and their association with their main dispersers, two short-tailed fruit bats, Carollia perspicillata and C. castanea (Phyllostomidae). Oikos 104(2): 362-376. doi: 10.1111/j.0030-1299.2004.12747.x

Werneck, M.S., M.E.G. Sobral, C.T.V. Rocha, E.C. Landau and J.R. Stehmann. 2011. Distribution and endemism of angiosperms in the Atlantic Forest. Natureza \& Conservação 9(2): 188-193. doi: 10.4322/natcon.2011.024

Author contributions: Amélia Carlos Tuler, inventory of Myrtaceae (data collection and identification); Eduardo de Sá Mendonça, writing and revision of the text, logistical and financial support; Filipe Torres, inventory of Rubiaceae (data collection and identification); Jaquelini Luber, floristics (data collection and species identification), data processing, drafting of images (plate), writing of the text; Jheniffer Abeldt Christ, inventory of Piperaceae (collection and identification); João Paulo Fernandes Zorzanelli, floristics (species identification); Karlo Gregório Guidoni-Martins, floristics (collection and identification), drafting of images (map) and writing of the text; Marcílio Zanetti, inventory of Rutaceae (collection and identification); Mário Luís Garbin, floristics (collection and identification), data processing and analysis, writing and revision of the text; Renan Köpp Holunder, floristics (collection and identification); Tatiana Tavares Carrijo, floristics (collection data and identification), data processing and analysis, writing and revision of the text; Vitor da Cunha Manhães, inventory of Bromeliaceae (collection and identification).

Received: 11 March 2015

Accepted: 22 January 2016

Academic editor: Juliana de Paula-Souza 